\title{
A genetic study of type 2 neurofibromatosis in the United Kingdom. II. Guidelines for genetic counselling
}

\author{
D G R Evans, S M Huson, D Donnai, W Neary, V Blair, V Newton, T Strachan, \\ R Harris
}

\begin{abstract}
The major defining features, age at onset of symptoms, and survival in 150 patients with type 2 neurofibromatosis (NF2) have been studied. The mean age at onset was 21.57 years $(n=110)$ and no cases presented after 55 years of age. Patients presented with symptoms attributable to vestibular schwannomas (acoustic neuroma), cranial meningiomas, and spinal tumours. In 97 cases studied personally by the authors, skin and eye examination were found to be useful to detect early signs of the condition. Examination of the skin is likely to assist in early diagnosis in at least $10 \%$ of cases and examination of the eye for a lens opacity or cataract in at least as many again. There are marked interfamilial differences in disease severity and tumour susceptibility. Vestibular schwannomas are not fully penetrant, but the condition is usually expressed in another way. Alteration to the current diagnostic criteria is advocated to cover the lack of provision for new mutations. A screening protocol is proposed and the effect of disease heterogeneity on management is discussed.

( $\mathcal{H}$ Med Genet 1992;29:847-52)
\end{abstract}

There has been an increasing awareness of the importance of differentiating NF1 and NF2 in recent years culminating in the mapping of the genes to different chromosomes ${ }^{1-4}$ in 1987 . In the same year the NIH Consensus Conference statement defined diagnostic criteria for NF1 and NF2. ${ }^{5}$ The NF2 criteria include distinct ophthalmological findings (posterior subcapsular lenticular opacities), although the frequency of these had been assessed in relatively small numbers of patients at that time. ${ }^{6}$ The NIH criteria for NF1 have been used in a large study and found to be satisfactory, ${ }^{7}$ but the NF2 criteria have not been similarly assessed.

A large clinical and genetic study of NF2 has allowed us to address these and other issues. The study has highlighted the inadequacy of current counselling and screening of at risk relatives. In this paper we present the findings of the study with relevance to genetic counselling, namely, the adequacy of current diagnostic criteria, the natural history of NF2, and suggestion of a screening protocol for at risk subjects.
Patients and methods

The methods of patient ascertainment, clinical assessment, and diagnostic criteria are described in the preceding paper. ${ }^{8}$ In addition all cases who did not precisely fit the NIH NF2 criteria (table 1), but who were thought to have the disease on clinical grounds were studied to allow evaluation of the diagnostic criteria.

\section{Results}

One hundred and fifty-five cases were entered into the study. After using additional criteria (table 2), five cases were considered to have insufficient criteria for the diagnosis of NF2; $120 / 150$ cases with NF2 had full clinical details available of whom 97 were personally studied by the authors. One family in which two cases had meningiomas, spinal and skin tumours and two isolated cases with a vestibular schwannoma and three and four meningiomas were included. Also included was one isolated case with multiple unilateral vestibular schwannomas and trigeminal neuroma, although she may represent segmental disease. The full details of the 120 cases in terms of their diagnostic criteria and presence of central nervous system tumours is presented in table 3. Cases of multiple meningiomas or multiple spinal tumours without vestibular schwannoma and with no skin or eye manifestations were excluded. Of the 150 cases, 110 are alive and mean age at death in the remaining 40 was 38.43 years (range 16 to 67 ).

\section{DIAGNOSTIC CRITERIA}

Cutaneous features

Skin examination in the 97 cases studied by the authors showed that only 40 cases $(41 \%)$ had

\section{Table 1 Diagnostic criteria for NF2.}

Bilateral vestibular schwannomas or family history of NF2 plus (1) unilateral acoustic or

(2) any two of: meningioma, glioma, neurofibroma schwannoma, posterior subcapsular lenticular opacities

Table 2 Revised diagnostic criteria for a diagnosis of NF2. The diagnosis of NF2 can be made in a subject who presents in any one of the following ways.

(1) NIH definition (table 1 ).

(2) Multiple meningiomata $(>1)+$ family history or a unilateral vestibular schwannoma, or at least 2 of: glioma, posterior subcapsular lenticular opacity, skin neurofibroma, or neurofibro

(3) Unilateral vestibular schwannoma $+>1$ meningioma or any 2 of glioma, posterior subcapsular lenticular opacity, skin neurofibroma, or neurofibroma or schwannoma of any cranial or peripheral nerve. 
Table 3 Diagnostic criteria, average age at onset, and death in 120 cases of NF2.

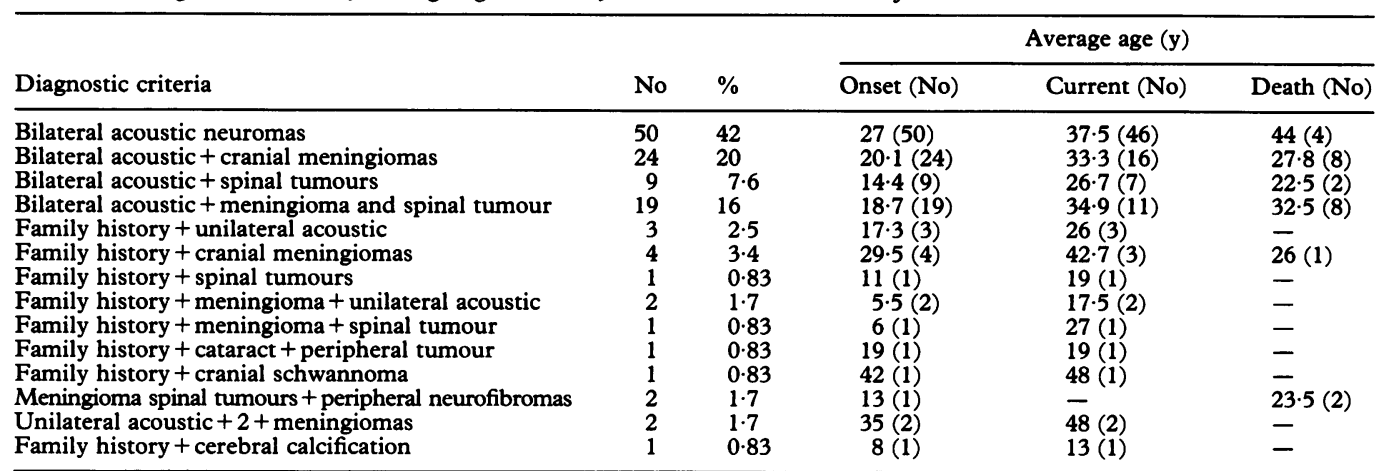

café au lait patches of greater than $0.5 \mathrm{~cm}$ in diameter before puberty or greater than $1.5 \mathrm{~cm}$ after puberty. Of these 21 had one patch, 11 had two, four had three, and three had four patches. Only one patient had six café au lait patches. Axillary and groin freckling was not convincingly found in any affected patient. Skin tumours were detectable in $66(68 \%)$ and varied in number from one to 27 . Only nine cases $(9 \%)$ had more than 10 skin tumours. They appeared clinically to be of three different types. The first and least common was similar to the papillary skin neurofibroma occurring in NF1 and was found in $27 \%$ of patients. The second type were deep to the skin and well circumscribed, often spherical tumours (fig 1); these occurred in $33 \%$ of patients. This type appeared to be occurring on peripheral nerves and the thickened nerve can sometimes be felt at either end of the tumour. The final and most common type first described by Martuza and Ojemann ${ }^{9}$ were discrete, slightly raised, roughened circumscribed areas of skin often pigmented and accompanied by excess hair; these were found in $47 \%$ of patients. No patient in this study had areolar neurofibromas or a plexiform neurofibroma. One case did, however, have an extracranial meningioma which had infiltrated the face and was clinically indistinguishable from a plexiform tumour.

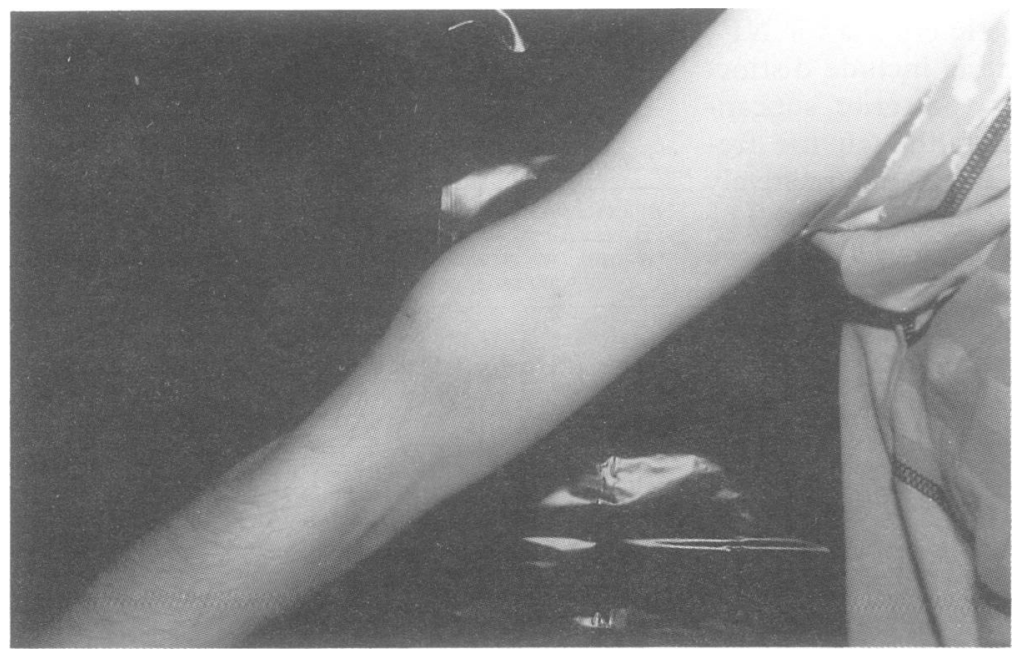

Figure 1 Subcutaneous deep nerve tumour in NF2. The thickened peripheral nerve was easily palpated at either end of the tumour.
Four of the 97 patients examined had noticed skin lumps as a prominent feature before any other symptom and three of these had them removed with a histological diagnosis of neurofibroma or schwannoma. Four out of the six patients remaining asymptomatic, but who had been diagnosed on scan as having acoustic tumours, had easily identifiable skin lumps. A further two cases, aged 27 and 52 respectively, who had a diagnosis of NF2 on the basis of a family history of NF2 plus meningiomas and spinal tumours, but who as yet had not developed vestibular schwannomas, had three and six skin tumours respectively. Therefore, in a total of $10 / 97$ cases skin examination would have aided early diagnosis.

\section{Overlap with NF1}

Only one patient in this series if seen in isolation could have fulfilled the diagnostic criteria for NF1 (table 3). This was the patient with six café au lait patches who also had two discrete skin tumours, although these were of the NF2 plaque type. The skin tumours in NF2 are far less numerous and usually clinically different from those occurring in NF1. Those that have been recently removed in patients with NF2 have been shown to be schwannomas and not neurofibromas.

\section{Ophthalmic signs}

Ninety cases were examined ophthalmologically and 57 have had slit lamp examination; 34 cases $(38 \%)$ have been identified as having lens opacities. Only four such patients were identified by slit lamp examination having been normal on ophthalmoscopy. Sixteen cases had been identified as having cataracts in the paediatric period and five cases had little vision in the affected eye from birth. The ophthalmic findings are the subject of a separate detailed study, but it appears that a congenital polar cataract also occurs in addition to the presenile posterior lenticular opacities previously noted. ${ }^{7}$ In addition to this, one case at $50 \%$ risk had a cataract associated with hyperplastic persistent primary vitreous, and another had anisometropic amblyopia, being blind in one eye without a cataract. A further four cases at $50 \%$ risk were identified as having large polar cataracts but were not included as affected 
cases as they had not yet developed other features.

Lisch nodules were not found to be a feature as only $2 / 55$ cases who had slit lamp examination exhibited one nodule which is probably within normal limits. ${ }^{10}$ No patients were shown to have retinal hamartomata.

Cataracts were known to be present before any other feature in $11 / 97$ cases personally examined. A further two cases from the 120 fully documented cases were known to have a cataract at the ages of 4 and 7 years, a full 19 and 11 years respectively before they became symptomatic. Thus a total of at least 13/120 cases were known to have a cataract, which eventually proved to be their first manifestation of NF2.

\section{CNS tumours and the NIH criteria}

There were four clear cases of familial NF2 who would not have fulfilled the NIH criteria at $52,63,26$, and 27 years of age, if they had been new mutations. The first case had multiple meningiomas, bilateral cataracts, and six skin tumours and a mother with bilateral acoustic tumours. The second case had multiple meningiomas only, with a daughter with bilateral vestibular schwannomas. The third case died from multiple meningiomas at the age of 26 years and has two daughters with bilateral vestibular schwannomas. The fourth case had a meningioma at the age of 5 years and a spinal tumour at 26 years and a father with bilateral vestibular schwannomas. None of these cases had vestibular schwannoma.

There were two sporadic cases with unilateral vestibular schwannoma plus three and four cranial meningiomas at the ages of 46 and 53 years respectively. Finally, a mother and son with multiple meningiomas and skin tumours, but no café au lait patches, died at the ages of 31 and 16 years, respectively, without vestibular schwannoma.

\section{MORBIDITY AND MORTALITY}

The average age at onset of symptoms in 110 cases in the UK was 21.57 years and at diagnosis 27.55 years. Mean age at death in 40 cases was 36.25 years, but including the living cases mean actuarial survival was 62 years.

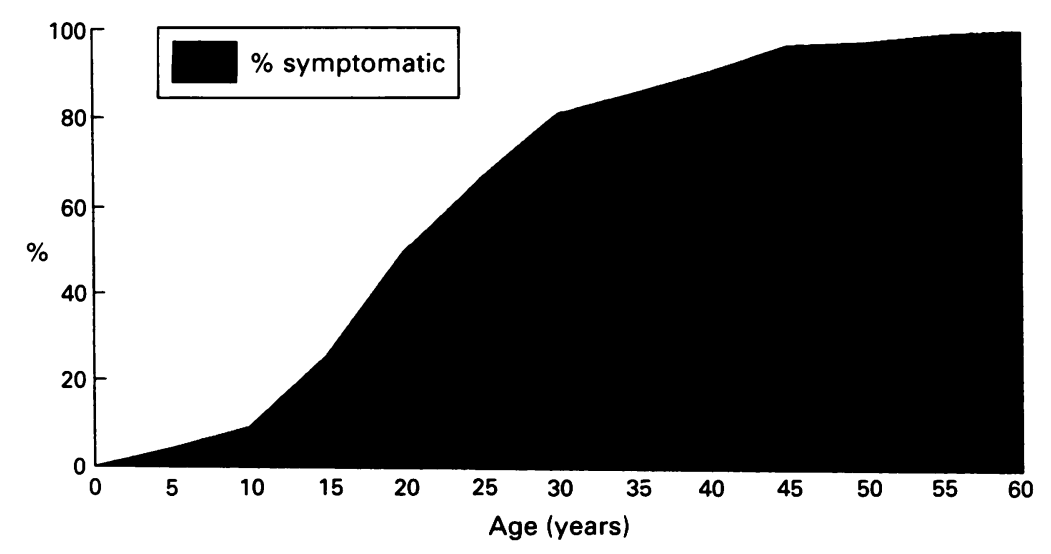

Figure 2 Cumulative incidence of 120 cases with NF2.
Mean actuarial survival from diagnosis was 15 years. In the North West region where there were 19 living cases, mean age at onset was 26.5 years and at diagnosis 30.5 years, showing that ascertainment bias was not causing identification of only severely affected cases from the remainder of the UK.

AGE AT ONSET CURVE

An age at onset of symptoms curve for 120 people with NF2 is shown in fig 2.

\section{EXPERIENCE OF THE FAMILIES}

Many of the families had little or no understanding of their condition though $11 / 19$ cases in the North West region had been referred for formal genetic counselling before the study; this compared to $33 / 91$ in the rest of the UK. Of those families not formally counselled, very few subjects had heard of neurofibromatosis or NF2. There was some degree of confusion with NF1 in those that had heard of neurofibromatosis, but this did not seem to be a confounding factor as these subjects knew it was the vestibular schwannomas that would be inherited. Many persons were completely unaware of the hereditary nature of the condition. Some form of screening of family members had been adopted in 43/75 families. This was comprehensive in $21 / 75$, in that annual to two yearly scans or brain stem evoked responses were offered to all those at $50 \%$ risk. This was reflected in the diagnosis of $11 / 110$ $(10 \%)$ of the cases asymptomatically. One of these cases had been shown to have abnormal brain stem evoked responses for two years with a normal CT scan and it was only when an MRI scan was performed that his acoustic tumour was visualised. Another case with abnormal audiology also had a negative CT scan before confirmation with MRI. Two subjects were diagnosed as a result of the study, but a further four cases with cataract at $50 \%$ risk may well go on to satisfy the criteria.

There was no clear evidence of a worsening of the disease during or just after pregnancy. One case had some transient tinnitus during pregnancy and another presented soon after pregnancy with deafness and tinnitus. Neither case had any serious sequelae and no intervention, surgical or otherwise, was required.

\section{Discussion}

This study has confirmed that the clinical presentation and natural history of NF2 is distinct from NF1. The main differences are the relative lack of peripheral features in NF2 and the fact that virtually all gene carriers develop tumours of the nervous system which carry significant morbidity and mortality. Finally, in NF2 severe disease features are limited to the nervous system in contrast to NF1 where complications can occur in almost any body system. ${ }^{6}$ Our study found no overlap with NF1 complications apart from nervous system tumours. The experience of the families in this study suggests there is a need for 
improving diagnostic awareness of NF2 and screening programmes for at risk relatives. The data presented here should help in achieving this goal.

In terms of diagnosis one of the main problems in the past has been the lumping together of cases with NF2 and NF1. For example, the study of Crowe $e t a l^{11}$ in 1956 concluded that $5 \%$ of subjects with NF1 have vestibular schwannomas. In retrospect, from the published clinical details it is clear these subjects had NF2. More recent studies, which clearly distinguish NF1 and NF2, have not shown an increased incidence of vestibular schwannomas in NF1 patients. ${ }^{612}$

Our study has shown that overlap in the clinical features of NF1 and NF2 rarely presents a diagnostic problem. In the 97 personally examined cases only one subject, whose mother had bilateral vestibular schwannomas, had six café au lait patches and two skin tumours (NF2 plaque type) on skin examination with asymptomatic bilateral vestibular schwannomas on MRI scan. With no family history and on examination by someone unaware of the different types of skin tumours, she could easily have been diagnosed as having NF1. There have been published reports of two similar cases, with no family history, recently..$^{913}$ In both reports the patients had bilateral vestibular schwannomas and in one more than six café au lait patches and skin neurofibromas including a plexiform tumour, and in the other six patches, multiple skin tumours, and a single Lisch nodule. A further case $^{14}$ clearly inherited NF1 from one parent and NF2 from the other. It is difficult to know whether the sporadic cases represent new mutations for both NF1 and NF2, or extreme variation in the skin features of NF2; in our cases no patient with NF2 has had a plexiform neurofibroma. This study has not given support to the designation of a third type of NF with features of both NF1 and NF2. ${ }^{12}$ The more common clinical situation, in our experience, is that patients with NF2 present for assessment of cutaneous features or symptomatic peripheral nerve tumours before developing symptomatic vestibular schwannomas and a diagnosis of NF1 is queried. The diagnosis of NF2 needs to be considered when subjects are referred for assessment with six or fewer café au lait spots and no other features of $\mathrm{NF} 1$, or when a young person presents with a spinal tumour, a meningioma, or a peripheral nerve tumour.

This study has confirmed that skin and eye examination is very useful in early diagnosis of NF2. The most comprehensive previous report found evidence of skin involvement in 19/ 31 cases. ${ }^{15}$ It is clear from our study that the peripheral nerve tumours precede the other major complications in at least $10 \%$ of cases. A detailed examination of the skin looking particularly for deep tumours on the nerve roots and discrete hairy macules in all at risk cases is, therefore, important. The discrete tumours are often best seen if a light source is shone at an acute angle to the skin. ${ }^{16}$ The more recently removed tumours for which pathology was available were schwannomas rather than neurofibromas and this again may be a way of distinguishing between the rare cases that manifest the criteria for NF1 and NF2.

Detailed eye examination is probably even more useful for early diagnosis, especially if a slit lamp examination is performed. The association between posterior subcapsular lens opacity and NF2 is only a recent finding. ${ }^{17}$ However, cataract has been noted to occur in cases as early as $1937 .{ }^{18}$ Unfortunately, not all the patients personally examined in this study have had a slit lamp examination, although over $30 \%$ had a lens opacity identifiable on ophthalmoscopy alone. The cataracts can be present at a very early age and may well be congenital. These earlier cataracts are polar rather than posterior subcapsular.

Other reports suggest that lens opacity may be present in up to $80 \%$ of NF2 cases $^{7}$ if slit lamp examination is performed. Cataracts had been identified as the first manifestation in $10 \%$ of our cases; therefore, a detailed ophthalmological examination of asymptomatic at risk subjects will show signs in a much greater proportion. There is also a strong case for examination of at risk neonates for congenital cataract, as if this is not identified, unilateral blindness is the likely sequel. No patient in this study had more than one Lisch nodule, which is probably within normal limits. ${ }^{10}$ There has been one report of multiple Lisch nodules in a patient with NF2, ${ }^{19}$ but our report does not support a suggestion that they are a feature of NF2 and we believe they are a useful distinguishing feature between the two types of NF. Retinal hamartomata have also been reported in $\mathrm{NF}^{20}$ and although none was found in this study, they may be a rare feature of the condition.

\section{HETEROGENEITY}

The NIH diagnostic criteria for NF2 (table 1) would have been satisfactory for the majority of cases in this study. There are, however, four cases in this study where, had they been new mutations, they would not have satisfied the diagnostic criteria. All four cases did not have vestibular schwannomas, but other members of these families did. To allow for these different presentations we suggest revision of the diagnostic criteria as presented in table 3 . These criteria should enable subjects with automosomal dominant multiple meningiomas (S Huson, A Harding, personal communications) or spinal schwannomas segregating as the only feature to be distinguished on clinical grounds. Molecular analysis with gene specific NF2 probes will allow us to address their genetic relationship in the near future.

The mode of presentation of NF2 within a particular family has an effect upon management. Surgery in any patient with NF2 is complicated and carries a higher morbidity and mortality than in patients with isolated vestibular schwannoma. ${ }^{21}$ In families with late onset vestibular schwannoma, disease progression is usually slow and a conservative approach may allow many more useful years of 
hearing. ${ }^{22}$ However, in patients presenting at a younger age with multiple, rapidly growing tumours a more aggressive approach is warranted. A cervical spine MRI scan is recommended in all cases before surgery, to avoid perioperative problems from asymptomatic cervical cord tumours. There is no convincing evidence that pregnancy worsens tumours already present in an NF2 case. Indeed the only report to show worsening of vestibular schwannoma in pregnancy ${ }^{23}$ showed no deterioration in the only NF2 patient over several pregnancies. Nevertheless, women with NF2 must be advised some caution before embarking on a pregnancy.

\section{GENETIC COUNSELLING AND SCREENING}

This study has shown a considerable delay between age of first symptom and diagnosis in NF2. Even in some at risk subjects the relevance of peripheral nerve tumours or congenital cataracts had not been appreciated. Only a minority of families had received adequate counselling and screening. The age at onset curve presented in fig 2 will aid genetic counselling. For example, for an asymptomatic at risk subject at 20 years of age their risk before screening of having inherited NF2 will have dropped to $25 \%$. As more families are followed prospectively a curve for age at diagnosis, with screening, will become available.

A protocol for screening subjects at risk of NF2 is shown in fig 3. The early age at onset with $10 \%$ of cases symptomatic before 10 years of age warrants earlier screening than generally suggested. The presence of congenital or very early cataracts necessitates detailed early ophthalmological screening and slit lamp evaluation should be used in addition to ophthalmoscopy at least every five years. Screening for vestibular schwannomas should begin in the early teens and brain stem evoked responses (BSER) should be performed annually. BSERs have been shown to be more useful in screening for vestibular schwannomas in NF2 than other audiological tests ${ }^{24}$; in at least two of our cases BSERs identified a vestibular schwannoma up to two years before a CT scan. Cranial imaging should be reserved for when an abnormality is detected on clinical or audiological examination and for counselling purposes. MRI scans are the imaging technique of choice, because they detect smaller tumours than CT and avoid the risk of radiation. A normal MRI scan at 16 to 18 years will approximately halve the risk of having inherited NF2 and will facilitate reproductive decision making. A normal scan at $\mathbf{3 0}$ years will make inheritance very unlikely except in late onset families and could lead to cessation of formal screening. Where onset has been late screening should be continued appropriately. With the mapping and then cloning of the gene, mutation studies will greatly enhance our knowledge of the condition and ability to counsel at risk subjects. Initially linkage studies will be an adjunct to the screening programme allowing cessation of testing at an earlier age in some cases. Eventually, however, some subjects will be able to be completely reassured if they are shown not to have inherited the family mutation in the NF2 gene and screening can be concentrated on asymptomatic high risk cases.

\section{Suspected patient}

Birth: detailed ophthalmic examination

Annual from 2-12: history, neurological and skin examination + ophthalmoscopy

Annual from 12-30: as before + audiological tests

At $16-18$ and 30 years or if suggestive screen: MRI scan

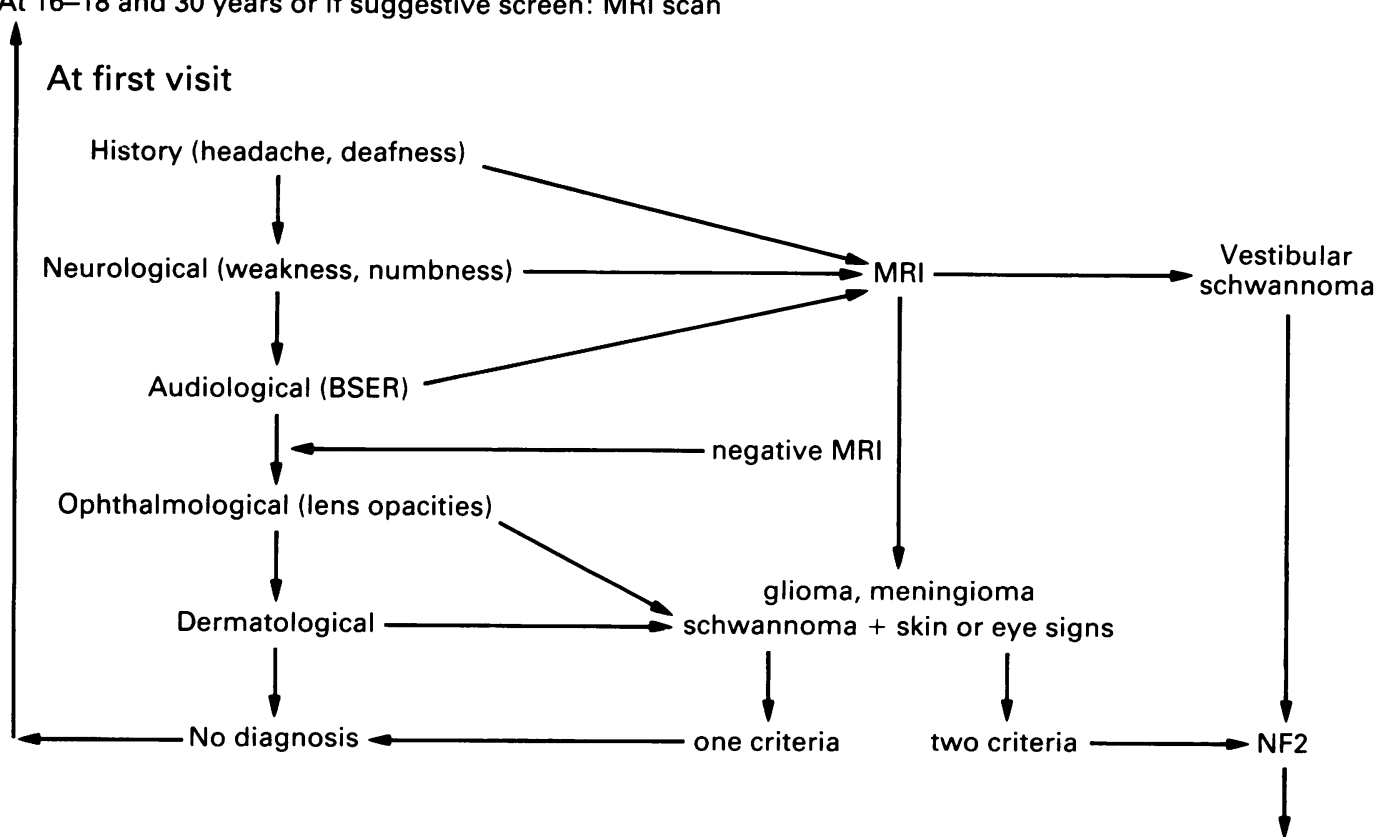

3-12 monthly appointments, regular MRI scans, operative procedures

Figure 3 Screening protocol for NF2. 
The surgical management of subjects with NF2 is complex and should probably be confined to specialist centres with experienced otolaryngologists and neurosurgeons. We have presented data elsewhere that show the subjects in this study treated at specialist centres had an improved outcome. ${ }^{21}$ As NF2 is a relatively rare condition, in a country the size of the UK perhaps only two or three such centres would be needed. Otolaryngologists, neurosurgeons, and clinical geneticists working together could coordinate the care and follow up of affected subjects and their at risk relatives. The cloning of the NF2 gene may provide insights into pathogenesis and suggest possible preventative treatments. Specialist centres with much experience in NF2 management would ensure clinical application of such developments, with careful appraisal, at the earliest opportunity.

We are grateful for the enormous help of clinicians throughout the UK who have supplied details of patients. We would particularly like to thank Mr T T King for supplying 19 cases and the Neurosurgical and Otolaryngology Units at the Manchester Royal Infirmary for their help in ensuring good ascertainment.

1 Seizinger BR, Rouleau GA, Ozelius LG, et al. Genetic linkage of von Recklinghausen neurofibromatosis to the nerve growth factor receptor gene. Cell 1987;49:589-94.

2 Barker D, Wright E, Nguyen $\mathrm{K}$, et al. Gene for von Recklinghausen neurofibromatosis is in the pericentromeric region of chromosome 17. Science 1987;236:1100-2.

3 Seizinger BR, Martuza RL, Gusella JF. Loss of genes on chromosome 22 in tumorigenesis of human acoustic neuroma. Nature 1986;322:644-7.

4 Rouleau G, Seizinger BR, Ozelius LG, et al. Genetic linkage analysis of bilateral acoustic neurofibromatosis to linkage analysis of bilateral acoustic neurofibromatosis to a. 8 .

5 National Institutes of Health Consensus Development Conference Statement on Neurofibromatosis. Neurofibromatosis Res Newsl 1987;3:3-6.

6 Huson SM, Harper PS, Compston DAS. Von Recklinghau- sen neurofibromatosis: a clinical and population study in south east Wales. Brain 1988;111:1355-81.

7 Kaiser-Kupfer MI, Freidlin V, Datiles MB, et al. The association of posterior capsular lens opacities with bilateral acoustic neuromas in patients with neurofibromatosis type 2. Arch Ophthalmol 1989;107:541-4.

8 Evans DGR, Huson S, Donnai D, et al. A genetic study of type 2 neurofibromatosis in the United Kingdom. I. Prevalence, mutation rate, fitness, and confirmation of maternal transmission effect on severity. $\mathcal{f}$ Med Gene 1992;29:841-6.

9 Martuza RL, Ojemann RG. Bilateral acoustic neuromas: clinical aspects, pathogenesis and treatment. Neurosurgery 1982;10:1-12.

10 Huson SM, Joned D, Beck L. Ophthalmic manifestations of neurofibromatosis. Br $\mathcal{f}$ Ophthalmol 1987;71:235-8.

11 Crowe FW, Schull WJ, Neal JV. A clinical, pathological and genetic study of multiple neurofibromatosis. Springfield, genetic study of multiple neurofibron

12 Riccardi VM, Eichner JE. Neurofibromatosis: phenotype, history and pathogenesis. Baltimore: Johns Hopkins University Press, 1986.

13 Michels VV, Whisnant JP, Garrity JA, Miller GM. Neurofibromatosis type 1 with bilateral acoustic neuromas. Neurofibromatosis 1989;2:213-7.

14 Sadeh M, Martinovits G, Goldhammer Y. Occurrence of both neurofibromatosis 1 and 2 in the same individual with a rapidly progressive course. Neurology 1989;39:2823.

15 Kanter WR, Eldridge R, Fabricant R, Allen JC, Koerber T. Central neurofibromatosis with bilateral acoustic neuroma: genetic, clinical and biochemical distinctions from peripheral neurofibromatosis. Neurology 1980;30:851-9.

16 Martuza RL, Eldridge R. Neurofibromatosis 2. $N$ Engl $f$ Med 1988;318:684-8.

17 Pearson-Webb MA, Kaiser-Kupfer MI, Eldridge R. Eye findings in bilateral acoustic (central) neurofibromatosis: findings in bilateral acoustic (central) neurofibromatosis: association with presenile lens opacity and cataracts but

18 Worster-Drought C, Dickson WEC, McMenemey WH Multiple meningeal and perineural tumours with analoMultiple meningeal and perineural tumours with analo-
gous changes in the glia and ependyma (neurofibroblasto-
matosis) Brain 1937;60:88-117.

19 Charles SJ, Moore AT, Yates JRW, Ferguson-Smith MA Lisch nodules in neurofibromatosis type 2. Arch Ophthalmol 1989;107:1571-2.

20 Good WV, Erodsky MC, Edwards MS, Hoyt WF. Bilatera retinal hamartomas in neurofibromatosis type $2 . \mathrm{Br}$ Ophthalmol 1991;75:190.

21 Evans DGR, Ramsden R, Harris R, Lye R, Huson SM, King TT. Type 2 neurofibromatosis: the need for supraregional care? f Laryngol Otol (in press).

22 Baldwin D, King TT, Chevretton E, Morrison AW. Bilateral cerebellopontine angle tumours in neurofibromatoteral cerebellopontine angle tumours in

23 Allen J, Eldridge R, Koerber T. Acoustic neuroma in the last months of pregnancy. Am $\mathfrak{f}$ Obstet Gynecol last months of

$24 \mathrm{NIH}$ conference statement. Neurofibromatosis 1 (von Recklinghausen disease) and neurofibromatosis 2 (bilateral acoustic neurofibromatosis). Ann Intern Med 1990;113:39-52. 